\title{
Novel imaging techniques to identify unstable arteriosclerotic plaques
}

Disruption of atherosclerotic plaques is the main cause for acute ischaemic syndromes. Up to now, the prediction of plaque rupture is challenging, since imaging techniques fail to precisely detect key characteristics of vulnerable arteriosclerotic plaques such as microcalcification, a large necrotic core, or positive remodelling. The development of modern functional molecular imaging techniques represent a novel approach to detect such unstable plaques in vivo, and therefore allows to identify patients at risk for cardiovascular events. There is increasing evidence for a potential role of PET-CT imaging in the diagnosis of cardiovascular disease. However the current gold standard, using ${ }^{18} \mathrm{~F}$-fluorodeoxyglucose $\left({ }^{18} \mathrm{~F}-\mathrm{FDG}\right)$ as a tracer does not allow for differentiation of plaque vulnerability, also ${ }^{18}$ F-FDG metabolically interferes with high blood glucose levels and therefore is not well applicable in patients with diabetes.

Joshi et al. investigated ${ }^{18} \mathrm{~F}$-sodium fluoride $\left({ }^{18} \mathrm{~F}-\mathrm{NaF}\right)$ as a new tracer for PET-CT imaging and its role in identifying vulnerable arteriosclerotic plaques in a prospective clinical trial on 80 patients with cardiovascular disease [1]. In detail, 40 patients with acute myocardial infarction (AMI) and 40 patients with stable angina pectoris underwent PET-CT as well as invasive coronary angiography. As tracer, ${ }^{18} \mathrm{~F}$-sodium fluoride $\left({ }^{18} \mathrm{~F}-\mathrm{NaF}\right)$ and ${ }^{18} \mathrm{~F}$-fluorodeoxyglucose $\left({ }^{18} \mathrm{~F}-\mathrm{FDG}\right)$, the current gold standard, were used. The tissue-to-background ratio as the uptake of ${ }^{18} \mathrm{~F}-\mathrm{NaF}$ in culprit and non-culprit lesions in AMI patients served as primary end-point. Secondary endpoints were comparative analysis of ${ }^{18} \mathrm{~F}-\mathrm{NaF}$ positive and negative atherosclerotic plaques with intravascular ultrasound imaging in patients with stable angina, and characterization of histological samples in symptomatic carotid artery disease.

The study found the highest ${ }^{18} \mathrm{~F}-\mathrm{NaF}$ uptake in culprit lesions of 37 (93\%) patients with AMI (median max. tissue-to-background ratio: culprit 1.66 [1.40-2.25] vs. highest non-culprit 1.24 [1.06-1.38]; $\mathrm{p}<0.0001$ ). However, standard PET-CT by use of ${ }^{18} \mathrm{~F}$-FDG did not discriminate between vulnerable and non-vulnerable plaques (1.71 [1.40-2.13] vs. 1.58 [1.28-2.01]; $\mathrm{p}=0.34)$. In stable angina, significant ${ }^{18} \mathrm{~F}-\mathrm{NaF}$ uptake occurred in $18(45 \%)$ patients. Further correlation with intravascular ultrasound showed more high-risk features in the ${ }^{18} \mathrm{~F}-\mathrm{NaF}$ marked lesions compared to those without: e.g. higher remodelling index (1.12 [1.09-1.19] vs. 1.01 [0.94-1.06];p = 0.0004), more microcalcification ( $73 \%$ vs. $21 \%$; $=0.002)$, or necrotic core $(25 \%$ vs. $18 \%$; $\mathrm{p}=0.001)$.

In summary, the use of non-invasive imaging techniques to detect ruptured or unstable atherosclerotic plaques would represent a seminal opportunity to identify patients at high risk for imminent cardiovascular events with further impact on treatment strategy. Looking at current literature, Adamson et al. assess PET-CT assisted plaque prediction favourably [2]. As a new tracer ${ }^{18} \mathrm{~F}-\mathrm{NaF}$ does allow for detection of high-risk plaques and may overcome some of the major drawbacks of the current standard method, using ${ }^{18} \mathrm{~F}-\mathrm{FDG}$ in terms of metabolic interaction. However, the method needs to be proven in a larger, non-selected patient cohort to give evidence for its broad applicability and predictive validity.

\section{References}

1. Joshi $\mathrm{N} V$ et al. ${ }^{18} \mathrm{~F}$-fluoride positron emission tomography for identification of ruptured and high-risk coronary atherosclerotic plaques: a prospective clinical trial. Lancet, 2014; 383: 705-13.

2. Adamson P et al. Translational Coronary Atherosclerosis Imaging with PET. Cardiol Clin 2016;34:179-86.

Jacqueline Stella \& Eva Freisinger

Division of Vascular Medicine, Department of

Cardiovascular Medicine

University Hospital Muenster, Muenster, Germany

Jaqueline.Stella@ukmuenster.de 